\title{
Factores asociados al éxito en programas para dejar de fumar
}

\author{
Moreno Arnedillo, J.J.(1); Herrero García de Osma, F.J.(2)
}

(1) Psicólogo. Centro de Prevención del Alcoholismo y Tabaquismo. Ayuntamiento de Madrid.

(2) Médico. Jefe de División. Centro de Prevención del Alcoholismo y Tabaquismo. Ayuntamiento de Madrid.

Enviar correspondencia a:

J. Javier Moreno Arnedillo. Centro de Prevención del Alcoholismo y Tabaquismo. Alberto Aguilera 20. 28015 MADRID. E-mail: arnedillo@correo.cop.es.

\section{RESUMEN}

Las bajas tasas de eficacia en los programas para dejar de fumar imponen la necesidad de investigar qué factores están asociados al éxito terapéutico. OBJETIVO: investigar el valor predictivo de distintos factores citados en trabajos anteriores, y establecer hipótesis sobre los mecanismos responsables de su valor predictivo. MATERIAL Y MÉTODOS: se toma una muestra de 130 participantes en el Programa para Dejar de Fumar del Ayuntamiento de Madrid, y se determina la significación estadística de las diferencias entre quienes permanecían sin fumar a los 6 meses y quienes habían recaído, en las variables objeto del estudio. RESULTADOS: se encuentran diferencias significativas en sexo, expectativas de autoeficacia y existencia o no de contactos con el tabaco durante las primeras semanas; se comparan con trabajos anteriores sobre estos y otros factores. CONCLUSIONES: 1) el sexo, las expectativas de autoeficacia y el no cometer "deslices" aparecen como los mejores predictores del éxito; 2) apostamos por un enfoque altamente individualizado de las estrategias terapéuticas para dejar de fumar.

Palabras clave: fumar, tabaco, dejar de fumar, predictores, tratamiento.

\section{INTRODUCCIÓN}

$\mathbf{F}$ umar tabaco es una conducta con un indudable valor instrumental. Con su uso, aparte de prevenir o aliviar los síntomas de abstinencia, se convierte en un potente y eficaz regulador del estado de ánimo y del comportamiento. Estimula cuando se está aburrido, y relaja cuando se está tenso. Sus efectos son casi inmediatos, es manejable, es bien tolerado socialmente, su administración es compatible con la mayoría de las actividades cotidianas, su uso se automatiza después de cientos de caladas-dosis diarias durante muchos años. Va acompañado de un ritual (sacar la cajetilla, extraer el cigarrillo, colocarlo en la

\section{SUMMARY}

The low efficacy rate of those programmes designed to cease smoking raises the need to investigate the factors linked to their therapeutical success. OBJECTIVE: To investigate the forecasting value of various factors that have been mentioned in previous studies, and to establish hypotheses about the mechanisms responsible for the forecasting value of those factors. MATERIALS AND METHODS: A sample of 130 participants in the "Programme to Stop Smoking" of Madrid's City Council is taken, and a statistical significance is determined between those who remained non-smokers six months later and those who had gone back to smoking, within the group of variables under consideration. RESULTS: Significant differences have been found with regard to gender, self-efficacy expectations and whether there has been, or not, any contact with tobacco during the first few weeks. They are compared with previous studies on these and other factors. CONCLUSIONS: 1/ Gender, self-efficacy expectations and the absence of slipping into old habits, all appear to be the best forecasters of success. 2/We bet on a highly individualized approach of the "stop smoking" therapeutical strategies.

Key words: smoking, tobacco, stop smoking, predictors, treatment.

boca, ofrecer, encenderlo...) que adquiere pronto, por condicionamiento pavloviano, la capacidad de provocar, por sí mismo, los efectos buscados en el cigarrillo. No es de extrañar que, propiedades bioquímicas de la nicotina aparte, su potencial adictivo sea enorme, acaso más potente que el de ninguna otra droga.

La investigación sobre el tabaquismo se orienta prioritariamente a establecer los procedimientos más idóneos para ayudar a dejar de fumar a quienes lo desean. Sin embargo, las bajas tasas de eficacia a largo plazo que se obtienen, en general, han llevado a plantear que, junto con la eficacia de las medidas terapéuticas per se, existen otras variables que condicionan poderosamente los resultados. Fumar es una conducta que aparece, se mantiene y en su caso se 
modifica como el resultado de la compleja y sutil interacción de factores individuales, factores del entorno y factores de la propia historia del hábito; la intervención terapéutica (e incluimos aquí los intentos de abandonar el tabaco por uno mismo, sin ayuda externa) es sólo un factor más que trata de modificar el comportamiento en una dirección, pero cuya potencia correctora es modulada por otras variables. No pretendemos afirmar que la técnica específica utilizada no sea relevante, pero tampoco olvidemos que los programas más eficaces informan de tasas de éxito en el tratamiento del tabaquismo que no exceden del $50 \%$ a los 6 meses, y eso cuando se trata de abordajes prolongados, multicomponentes y con individuos motivados.

Más aún: incluso entre los factores terapéuticos está sobradamente demostrada la importancia de los aspectos inespecíficos, hasta el punto de que en algunos trabajos (Froján, 1991) no se encuentran diferencias significativas en los resultados entre los grupos experimentales y los grupos de control. Como apunta esta autora (y nosotros corroboramos), el mero hecho de apuntarse a un programa para dejar de fumar, de asistir periódicamente a sesiones en grupo o de completar autorregistros puede ser suficiente para conseguir y mantener la abstinencia, dadas ciertas condiciones individuales. Y, para completar el cuadro, recordemos que el porcentaje de exfumadores que abandonaron su hábito por su cuenta, sin ayuda profesional, supera el 90\% (Fiore et al., 1990; García Merino, 1998).

Son numerosos los factores que se han relacionado con los resultados:

- Factores del individuo: características individuales, repertorios conductuales, aspectos motivacionales, etc. (Nides et al., 1995; Swan et al., 1988; Norregaard et al., 1993; Bjornson et al., 1995; Mankani et al., 1996; Ward et al., 1997; Blake et al., 1989; Gourlay et al., 1994; Matheny y Weatherman, 1998; Garvey et al., 1992; Vázquez y Becoña, 1998; Scholte y Breteler, 1997; Kenford et al., 1994; Westman et al., 1997; Kamarck et al., 1988; Hughes et al., 1992).

- Factores del contexto: si existe apoyo familiar, si los compañeros de trabajo fuman, si existe estabilidad laboral, cómo son los recursos sanitarios, etc. (Daughton et al., 1990; Matheny y Weatherman, 1998; Secker-Walker et al., 1990).

- Aspectos formales o inespecíficos de la intervención: si la terapia es en grupo o individual, qué periodicidad tienen las sesiones, cuánto dura el tratamiento, si la intervención se desarrolla en una clínica privada y de pago o en un centro público de salud o en la empresa, etc.

- Factores de la relación con el tabaco: qué intentos previos han existido, en qué fase del proceso de cambio se encuentra el sujeto, qué grado de confianza manifiesta sobre su capacidad para mantenerse sin fumar, etc. (Stuart et al., 1994; Haaga y Stewart, 1992; Scholte y Bleteler, 1997; Kowalski, 1997; Gulliver et al., 1995; Grove, 1993; Garvey et al., 1992; Secker-Walker et al., 1990; Farkas et al., 1996; Vázquez y Becoña, 1998).

Este trabajo pretende exponer los hallazgos obtenidos sobre los factores asociados en el éxito en intentos de dejar de fumar a partir del Programa para Dejar de Fumar del Ayuntamiento de Madrid, actualmente en marcha y dirigido al colectivo de funcionarios de esta institución (aproximadamente 18.000 personas).

El estudio trata de replicar hallazgos anteriores sobre los factores sociodemográficos, conductuales y de relación con el tabaco (variables independientes) que permiten predecir la evolución de la cesación tabáquica a los 6 meses de finalizado el tratamiento (variable dependiente).

A partir de la revisión bibliográfica, del modelo teórico de partida (Teoría del Aprendizaje Social) y de la observación no sistematizada, se seleccionaron las siguientes variables y se estimó su valor predictivo en la dirección que se indica.

\section{A. FACTORES DE LA RELACIÓN CON ELTABACO.}

\section{- Severidad de la dependencia: número de cigarri- llos diarios, puntuación en el test de Fagerstrom, contenido en nicotina del tabaco consumido.}

Se hipotetiza mejor pronóstico para los individuos con indicadores más bajos de dependencia. Vázquez y Becoña (1998) encuentran tanto el número de cigarrillos como el contenido en nicotina de los mismos como variables predictivas de la abstinencia en un seguimiento de 12 meses. Otras investigaciones encuentran mejores resultados en individuos con puntuaciones más bajas en el test de Fagerstrom (Norregaard et al., 1993; Westman et al., 1997; Daughton et al., 1990; Curry et al., 1989). Sin embargo, existen trabajos que no encuentran valor predictivo en este conjunto de variables (Kenford et al., 1994; Matheny y Weatherman, 1998).

\section{- Historia de consumo: años fumando, intentos previos para abandonar el tabaco, duración de los períodos previos de abstinencia.}

Se hipotetiza mejor pronóstico para los individuos que llevan más años fumando, que han llevado a cabo uno o dos intentos previos y en los que la duración de estos intentos previos es más larga, por el efecto que esta experiencia puede producir en las expectativas de 
autoeficacia. Barnes et al. (1985) encuentran predictiva de abstinencia la existencia de pocos intentos previos; Garvey et al. (1992) encuentran que la existencia de varios intentos previos de corta duración disminuyen significativamente la probabilidad de mantener la abstinencia. Secker-Walker et al. (1990) encuentran una correlación significativa entre la abstinencia a los 6 meses y la duración de los intentos previos.

\section{- Existencia de consumos aislados ("deslices") durante las primeras semanas del tratamiento.}

Se hipotetiza mejor pronóstico para los individuos que no han experimentado ningún contacto con el tabaco en las primeras semanas. Kenford et al. (1994), en una investigación sobre 200 sujetos sometidos a dos condiciones de tratamiento con parches de nicotina, no encontraron correlación con los resultados a los 6 meses para ninguna variable de severidad de la dependencia (puntuación en el test de Fagerstrom, número de cigarrillos diario, años fumando, nivel de CO, severidad del síndrome de abstinencia...). Sí encontraron, por el contrario, un sólido valor predictivo en los llamados "deslices" (consumos aislados de algún cigarrillo o incluso sólo de alguna calada) durante las dos primeras semanas de tratamiento: el $83 \%$ y el $97 \%$ (en cada una de las dos condiciones experimentales) de los que habían cometido un "desliz" durante las dos primeras semanas estaban fumando a los 6 meses; por el contrario, sólo el $46 \%$ y el $41 \%$ de los que mantuvieron totalmente la abstinencia en las dos primeras semanas estaban fumando a los 6 meses. Nides et al. (1995), con una muestra de 3.923 participantes en un estudio sobre salud pulmonar donde se recibía tratamiento cognitivo-conductual para dejar de fumar, encontraron que el mejor predictor de la recaída entre los 4 y los 12 meses después de abandonar el tabaco era precisamente haber fumado al menos un cigarrillo entre el primer día y los 4 meses del abandono. Tratando de afinar más aún, Westman et al. (1997) encuentran que la probabilidad de abstinencia a los 6 meses de abandonar el tabaco está significativamente asociada a la abstinencia total en el primer día de dejar de fumar: los individuos abstinentes durante el primer día tenían 4 veces más probabilidades de estar abstinentes a los 6 meses que los individuos que habían fumando algo. Otros trabajos realizados a partir de ensayos clínicos (Norregaard et al., 1993; Kamarck et al., 1988) o sobre individuos que habían hecho intentos por su cuenta (Hughes et al., 1992; Garvey et al., 1992; Ward et al., 1997) encuentran invariablemente resultados similares.

\footnotetext{
- Entorno social en relación con el tabaco: presencia de fumadores en la familia o en el lugar de trabajo.
}

Se hipotetiza mejor pronóstico para los individuos con menor presencia de fumadores en el entorno. Algunos trabajos (Daughton et al., 1990; Matheny y Weatherman, 1998; Secker-Walker et al., 1990) encuentran valor predictivo en el número de fumadores que conviven en el domicilio, o en la percepción subjetiva de apoyo por parte de la pareja. Existe también algún dato (Collins et al., 1990) que apunta a que el apoyo de los compañeros de trabajo, o la existencia de un entorno laboral con fumadores es un factor más predictivo que el apoyo de la pareja (Klesges et al., 1988; Garvey et al., 1992; Havassy et al., 1991). Richmond et al. (1988) y Nides et al. (1995) encuentran que los resultados correlacionan significativamente con el número de compañeros de trabajo que fuman o con el apoyo percibido en el lugar de trabajo o en el entorno social en general.

\section{- Motivación para dejar de fumar: presencia de sín- tomas leves o graves asociados al tabaco, predis- posición al cambio según el modelo transteórico de Prochaska y Prochaska (1993).}

Se hipotetiza mejor pronóstico para aquellos individuos que presentan mayor número de síntomas asociados al consumo de tabaco, y que se declaran en situación de "preparación" para el abandono. El modelo de las fases del proceso de cambio de Prochaska et al. (1993) predice que la probabilidad de que el cambio en la conducta adictiva se produzca y se mantenga es mayor en los individuos que se encuentran en fase de "preparación" que en aquellos que están en fase de "contemplación", y mayor aún que en los individuos en fase de "precontemplación". Esta predisposición al cambio sería función de dos variables: por un lado, la naturaleza y el peso de las motivaciones que presenta el individuo para dejar de fumar; por otro, las expectativas de autoeficacia. Ambas variables son más consistentes en los "preparados" y "contempladores" que en los "precontempladores" (DeVries et al., 1998): los primeros perciben más ventajas en abandonar el tabaco, y se sienten más capaces de conseguirlo que los últimos, por lo que esta variable sería predictiva de los resultados. Secker-Walker et al., (1990) así lo encuentran cuando se compara a los individuos en fase de "preparación" (definida operativamente como "fuerte intención de dejarlo en el próximo mes") frente al resto. Sin embargo, Farkas et al. (1996) consideran que otras variables son mejores predictores.

\section{- Incremento de peso al dejar de fumar.}

Se hipotetiza mejor pronóstico para los individuos que no aumentan de peso durante el tratamiento, o que manifiestan menor preocupación por el posible aumento de peso. Norregaard et al. (1993) encuentran 
que los sujetos que ganan peso durante las primeras semanas mantienen altas tasas de éxito, mientras que los que ganan peso después de 3 meses presentan mayor tasa de recaídas. Cuando consideramos no la ganancia real de peso o el momento en que se produce, sino la preocupación previa por ganar peso, parece ser que sí se puede predecir mejor la recaída o el no intento de abandono (Klesges et al., 1988). Pinto et al. (1999), sobre una muestra de 281 mujeres, sí encuentran que el control del peso es un importante reforzador de la conducta de fumar dadas ciertas condiciones: mujeres jóvenes, con puntuaciones altas en el test de Fagerstrom, que han ganado peso en intentos anteriores y que anticipan que ganarán peso si dejan de fumar. Gourlay et al. (1994), sobre una muestra de 1481 sujetos, encuentra también asociaciones significativas entre la preocupación por ganar peso y la probabilidad de recaída.

\section{B. FACTORES PSICOLÓGICOS}

\section{- Autoeficacia percibida para mantenerse sin fumar.}

Se hipotetiza mejor pronóstico para los individuos que puntúan más alto en una escala de autoeficacia. A partir de la Teoría del Aprendizaje Social (Bandura, 1977) en su aplicación al campo de las conductas adictivas (Annis, 1987) se asume que la eficacia del proceso de afrontamiento del deseo de fumar depende de dos variables clave íntimamente asociadas: el repertorio de respuestas de afrontamiento, y las expectativas de autoeficacia. El repertorio de respuestas de afrontamiento es el conjunto de habilidades conductuales y cognitivas con que cuenta el individuo para afrontar las situaciones asociadas al consumo (como habilidades sociales o habilidades de autocontrol emocional). Las expectativas de autoeficacia se refieren a las creencias del individuo sobre su capacidad para afrontar con éxito (es decir, sin consumir) esas situaciones. Ambas variables correlacionan positivamente con la abstinencia: invertimos más esfuerzo en conseguir un objetivo cuando nos sentimos capaces de alcanzarlo y además contamos con las destrezas necesarias. Por lo tanto, dos de los objetivos terapéuticos centrales son el incremento del repertorio de habilidades de afrontamiento, y el incremento de las expectativas de eficacia. Es importante señalar que las expectativas de eficacia dependen fundamentalmente de la ejecución exitosa de la conducta: ejecuciones exitosas aumentan nuestra autoconfianza y nuestras expectativas de éxito futuro; ejecuciones fracasadas las disminuyen. De este principio se deriva el papel terapéutico central de las técnicas de exposición al estímulo: el objetivo central del tratamiento sería incrementar las expectativas de autoeficacia del individuo, como mejor predictor de la abstinencia futura; dado que la fuente principal de las expectativas de autoeficacia es la propia ejecución exitosa, el tratamiento debe dirigirse a someter al individuo a tareas de afrontamiento real en situaciones de riesgo, con el fin de incrementar su percepción de competencia; por supuesto, es crítico que la probabilidad de éxito en la tarea de afrontamiento sea elevada, y para ello pueden utilizarse distintos procedimientos.

La hipótesis sobre el poder predictivo de esta variable ha sido sometida repetidamente a contrastación, con resultados favorables (Stuart et al., 1994; Haaga y Stewart., 1992; Scholte y Breteler, 1997; Kowalski, 1997; Gulliver et al., 1995; Grove, 1993; Garvey et al., 1992), si bien se van definiendo algunos matices:

- La expectativas de autoeficacia para mantenerse sin fumar, como manifestación subjetiva, son el resultado de distintos determinantes, aparte del afrontamiento exitoso pasado: el estado de ánimo (de ahí el mal pronóstico de los individuos deprimidos), el repertorio de respuestas de afrontamiento, la atribución de los fracasos anteriores, la experiencia vicaria o la generalización de las expectativas de autoeficacia en otras áreas.

-La autoeficacia es predictiva cuando ya se lleva un tiempo sin fumar, es decir, cuando se basa en la propia ejecución exitosa. En nuestra muestra, sólo resultó predictiva la medida de autoeficacia tomada a los 3 meses de finalizado el tratamiento. Tampoco Mothersill et al. (1988) encuentran predictiva la medida inicial de la autoeficacia.

- La autoeficacia correlaciona positivamente con el éxito en el mantenimiento de la abstinencia, pero negativamente con los intentos de abandono (Stuart et al., 1994). Al parecer, la propia seguridad del "puedo dejarlo cuando quiera" Ileva a aplazar la decisión de dejar de fumar.

- La relación entre la autoeficacia y la probabilidad de éxito parece tener forma de $U$ invertida: el nivel idóneo de autoeficacia sería un nivel alto, en el que si ocurre un consumo aislado el individuo no pierde la esperanza de seguir sin fumar, pero no tan alto que induzca al individuo a experimentar con el tabaco y probablemente a recaer (Haaga y Stewart, 1992).

\section{FACTORES DEMOGRÁFICOS.}

\section{- Sexo.}

Se hipotetiza mejor pronóstico para los varones. De once investigaciones revisadas, en ocho se encuentran diferencias significativas en esta dirección (Nides et al. (1995);, Swan et al. (1988), Norregaard et al. (1993);, Bjornson et al. (1995); Mankani et al. (1996); Ward et al. (1997); Blake et al. (1989), Gourlay 
et al. (1994)). Debe notarse que en tres trabajos no es así: el de Matheny y Weatherman (1998), el de Garvey et al. (1992) y especialmente, por haber sido realizado en nuestro país y con un seguimiento de 12 meses, el de Vázquez y Becoña (1998).

La explicación de esta diferencia ha dado lugar a distintas hipótesis. En principio, caben al menos dos posbilidades simples: en primer lugar, los programas estarían diseñados para características del consumo de tabaco y para recursos de autocontrol más frecuentes en el varón; en segundo lugar, las mujeres se mostrarían más proclives a participar en programas para dejar de fumar (lo cual en nuestro caso es cierto), lo que supondría un plus de motivación en los varones que deciden participar. Sin embargo, Ward et al. (1997) siguen encontrando diferencias significativas en una muestra formada por individuos que abandonan el hábito por sí mismos (94\% del total de los exfumadores, Fiore et al., 1990); en este mismo trabajo no se encuentran diferencias entre sexos en factores como edad, historia del consumo, apoyo social, estrés y motivación, que pudieran ser responsables de las diferencias observadas. Blake et al. (1989), sobre una muestra de 1669 fumadores actuales, encuentran también que los intentos previos de dejar de fumar y las expectativas de conseguirlo son menos consistentes en el caso de las mujeres. Epstein et al. (1984) aporta datos para una hipótesis que explicaría las diferencias entre ambos sexos en la función que cumple la conducta de fumar. Sabemos que fumar puede actuar como un regulador del estado de ánimo de distintos modos en función de los individuos, los contextos y las expectativas. Puede producir un efecto estimulante cuando se está aburrido, o calmante cuando se está tenso. Swan et al. (1988) encontraron que la recaída se asociaba a historia familiar y estrés en el caso de los hombres, y a historia familiar, estrés, características del fumar, actividad física y variables relacionadas con el trabajo en el caso de las mujeres. Ikard y Tomkins (1973) encontraron que las mujeres declaran que fuman para reducir un estado anímico negativo como ansiedad o ira en un porcentaje mucho mayor (73\% frente a $36 \%$ ) que los hombres; estos, por el contrario, declaran con más frecuencia que fuman buscando estimulación y placer. Ello se debería a que el descenso en la percepción subjetiva de tensión muscular que se produce al fumar en situaciones de alta excitabilidad es mayor en las mujeres (Epstein, 1984), lo que explicaría la diferente relación funcional de la conducta de fumar en hombres y en mujeres. En términos de teoría del aprendizaje, el mantenimiento de la conducta de fumar se produciría más por refuerzo negativo en el caso de las mujeres, y más por refuerzo positivo en el caso de los hombres. De ahí la mayor dificultad de aquellas para su abandono (Russell et al., 1974). La diferencia observada en las expectativas de autoeficacia entre sexos sería la manifestación subjetiva de este fenómeno.

\section{- Edad.}

Se hipotetiza mejor pronóstico para los individuos de más edad. Gourlay et al. (1994) encuentran diferencias significativas favorables a los individuos de más de 40 años. Garvey et al. (1992), por otro lado, no las encuentran, si bien la muestra de este último estudio es bastante más reducida. Probablemente la edad es una variable que está menos relacionada con los resultados, y más relacionada con la motivación y la realización de intentos consistentes por dejar de fumar.

Existen dos variables relevantes que no han sido incluidas en el estudio, y que merecen una breve revisión en esta introducción por haber resultado predictivas en distintos trabajos: la existencia de problemas de depresión o ansiedad, y el ejercicio físico.

Es abundante la investigación que trata de dilucidar la relación entre el mantenimiento del abandono y la frecuencia e intensidad de estados emocionales, principalmente ansiedad y depresión, o distintos factores que mediatizan su probabilidad de aparición, como el repertorio de habilidades de afrontamiento o el valor de dimensiones de personalidad como "neuroticismo" o "extroversión". En general, esa línea de investigación ha resultado sumamente productiva. Está bien establecida la mayor incidencia de estados depresivos entre los fumadores que entre los no fumadores (Bayot y Capafons, 1995), y el papel de la nicotina como elemento regulador del estado de ánimo (Bock et al., 1996) y por tanto el mantenimiento de la conducta de fumar por reforzamiento negativo al aliviar o evitar la disforia. De modo coherente con estos datos, Covey et al. (1990) y Ginsberg et al. (1997) encuentran que la historia previa de depresión aumenta la probabilidad de aparición de conductas depresivas tras el abandono del tabaco, y que ello condiciona poderosamente los resultados. Vázquez y Becoña (1998), por su parte, encuentran la menor sintomatología depresiva como la única variable predictiva de la abstinencia a los 12 meses. Así mismo, se ha encontrado de modo consistente un peor pronóstico en fumadores que puntúan alto en medidas de ansiedad y estrés (Curry et al., 1989; Swan et al., 1988; Gulliver et al., 1995), así como menos intentos de abandonar el tabaco por parte de individuos que fuman para afrontar el estrés (Zimmerman et al., 1990). En esta misma línea, Kamarck y Lichtenstein (1988) y Matheny y Weatherman (1998) encuentran predictivas del mantenimiento de la abstinencia las medidas de competencia social y de autocontrol emocional.

En cuanto al ejercicio físico, la relación entre esta variable y el abandono del tabaco ha sido encontrada en repetidas ocasiones. Swan et al. (1988) encontraron que la actividad física correlacionaba con la abstinencia particularmente en mujeres y de modo menos significativo en hombres. King et al. (1996) encontraron, en relación con el dato anterior, que los fumado- 
res que practican ejercicio regularmente manifiestan significativamente más confianza en su capacidad para mantenerse sin fumar. Probablemente tanto el dejar de fumar como el practicar ejercicio son dos pautas de conducta que están mediadas por los mismos mecanismos motivacionales (adoptar un estilo de vida más saludable) y conductuales (expectativas de autoeficacia). Ello explicaría que los cambios en una conducta correlacionen con cambios en la otra. Pero aparte de los ingredientes motivacionales y conductuales comunes que explicarían el mejor pronóstico de los fumadores que practican o están dispuestos a practicar ejercicio físico, parece que éste último, en sí mismo, favorece el mantenimiento de la abstinencia. Marcus et al. (1999), sobre una muestra de 281 mujeres sedentarias, encuentran que aquellas que habían sido asignadas aleatoriamente a una condición de tratamiento cognitivo-conductual que incluía tres sesiones de ejercicio físico supervisado por semana conseguían una abstinencia continuada significativamente superior al grupo idéntico pero que no incluía ejercicio físico, tanto al final del tratamiento como a los 3 y 12 meses, concluyendo que la práctica de ejercicio mejora la capacidad vital y atenúa la ganancia de peso que sigue a dejar de fumar. Probablemente (añadimos nosotros) ofrece además una oportunidad más evidente de percibir la mejoría física y refuerza la motivación.

\section{MATERIALY MÉTODOS}

\section{Muestra}

A partir de un conjunto de 155 individuos que inician el tratamiento, la muestra está formada por 130 individuos que han completado, hasta la fecha, el Programa para Dejar de Fumar del Ayuntamiento de Madrid, y cuyos datos, hasta el seguimiento de 6 meses, están completos. No se tienen en cuenta, por lo tanto, a las personas que abandonaron el programa o que se perdieron a lo largo del seguimiento, ni tampoco a las que están en tratamiento o seguimiento en el momento de redactar este estudio.

El programa está descrito detalladamente en Moreno y Herrero (1998), y sus resultados provisionales en Herrero, Moreno y Rivero (en preparación). Se trata de un programa dirigido al colectivo de trabajadores del Ayuntamiento de Madrid, con una población mayoritariamente sana, con participación totalmente voluntaria y cuyas sesiones tienen lugar dentro del horario laboral. En una primera fase, la que abarca el presente estudio, la intervención se centró casi exclusivamente en el personal del Area de Salud y Consumo. El programa tiene una duración de 8 sesiones semanales, de 1,5 horas de duración, y siempre en formato de grupo de entre 8 y 14 personas. Está conducido por un psicólogo, un médico y un enfermero. Sus ingredientes terapéuticos son:

- Técnica específica para dejar de fumar. Se da la opción de elegir entre la técnica de reducción gradual de la ingesta de nicotina mediante el cambio de marca (Becoña, 1994) o el uso de parches de nicotina transdérmica, cuyo coste es financiado por la propia institución.

- Técnicas de motivación.

- Técnicas de autocontrol y prevención de recaídas (control estimular, relajación, identificación de excusas para fumar, fomento de hábitos alternativos, control del efecto de violación de la abstinencia...).

En cuanto a las características sociodemográficas de la muestra, un $31,4 \%$ son varones y el 68,6\% mujeres; la media de las edades es 40,36 años y la desviación típica 7,43; todos ellos son trabajadores en activo del Ayuntamiento de Madrid; un 36\% son titulados superiores (mayoritariamente médicos y psicólogos), un $23,1 \%$ son diplomados (enfermeros $y$ trabajadores sociales), un 6,9\% poseen titulación de FP2 o equivalente, y un 27,9\% poseen titulación de FP1. Se trata, por lo tanto, de una muestra especial, propia de un programa llevado a cabo en una empresa, con pleno empleo, con un nivel profesional elevado y con un grado de conocimiento sobre la problemática del tabaquismo muy alto.

En cuanto a datos del consumo de tabaco, hay que destacar que, salvo excepciones, se trata de una muestra sana, donde sólo 4 sujetos declaraban más de dos problemas severos de salud asociados al tabaco. La media de consumo era de 27,35 cigarrillos diarios, con un rango entre 5 y 55 y una desviación típica de 10,64. La media de años fumando era de 21,9 con un rango entre 2 y 46 y una desviación típica de 6,82. La puntuación media en el test de Fagerstrom es 5,17, con una desviación típica de 2,45. El número de intentos previos de abandonar el tabaco variaba en un rango entre 0 y 5 , con una media de 1,11 . En los casos en que había existido algún intento previo de abandonar el tabaco, el período más largo de abstinencia era, de media, 29,77 semanas, con una desviación típica de 44,79 semanas. Un 53,6\% de los sujetos declaraban que su deseo de dejar de fumar era "total", y un $36,2 \%$ que era "bastante grande". En cuanto al contenido de nicotina y alquitrán del tabaco, un 63\% fumaba una marca "normal", un 32,7 fumaba una marca "light" y un 4,2 fumaba una marca "ultralight."

\section{Evaluación}

Los datos de evaluación inicial, fisiológica y conductual, así como los instrumentos utilizados, aparecen en la tabla 1. 


\section{Tabla 1: Variables e instrumentos de evaluación}

\begin{tabular}{|c|c|}
\hline VARIABLES E INSTRUMENTO & EVALUACIÓN \\
\hline TÉCNICA UTILIZADA & DATOS RECOGIDOS \\
\hline Analítica de sangre & $\begin{array}{l}\text { - Parámetros de laboratorio alterados por el hábito tabáquico. } \\
\text { Principalmente: } \\
\text {-Hematocrito. } \\
\text {-Hemoglobina. } \\
\text {-Volumen corpuscular medio. } \\
\text {-Hemoglobina corpuscular media. } \\
\text {-Concentración de hemoglobina corpuscular media. }\end{array}$ \\
\hline Espirometría & $\begin{array}{l}\text { - CVF. } \\
\text { - VEF1. } \\
\text { - PEF. } \\
\text { - FEF25-75. } \\
\text { - Vmáximo } 25 . \\
\text { - Vmáximo } 50 . \\
\text { - Vmáximo } 75 .\end{array}$ \\
\hline Cooximetría & - Concentración CO en aire espirado. \\
\hline $\begin{array}{l}\text { Inventario de síntomas de } \\
\text { abstinencia }\end{array}$ & $\begin{array}{l}\text { - Registro diario de la existencia e intensidad de síntomas de } \\
\text { abstinencia, durante las semanas siguientes a dejar de fumar. }\end{array}$ \\
\hline Test de Fagerstrom & - Severidad de la dependencia \\
\hline $\begin{array}{l}\text { Cuestionario sobre el Hábito de } \\
\text { Fumar (adaptado de Becoña, } \\
\text { 1994) }\end{array}$ & $\begin{array}{l}\text { - Datos demográficos. } \\
\text { - Historia del consumo de tabaco. } \\
\text { - Consumo actual de tabaco: número de cigarrillos, intensidad } \\
\text { (inhalación, número de caladas, cantidad fumada). } \\
\text { - Presencia de fumadores en el entorno. } \\
\text { - Intentos previos para dejar de fumar o fumar menos } \\
\text { (cuántos, cuánto tiempo, por qué medios). } \\
\text { - Motivos para dejar de fumar. } \\
\text { - Nocividad que atribuye al tabaco. } \\
\text { - Síntomas, actuales o pasados, atribuibles al tabaco. } \\
\text { - Consumo de alcohol, café y medicamentos. } \\
\text { - Grado de deseo de dejar de fumar en la actualidad. } \\
\text { - Expectativas sobre el tratamiento. }\end{array}$ \\
\hline $\begin{array}{l}\text { Escala de Confianza en } \\
\text { Situaciones de Fumar }\end{array}$ & $\begin{array}{l}\text { - Grado de autoeficacia percibida para mantenerse sin fumar, } \\
\text { global y situacional. }\end{array}$ \\
\hline
\end{tabular}

ladaptado de Baer y

Lichtenstein, 1988)

La evaluación final, así como la evaluación de seguimiento a los 3 y 6 meses, se realizó a través del cuestionario de seguimiento de Becoña (1994) y el cuestionario de autoeficacia adaptado de Baer y Lichtenstein (1998) (Moreno y Herrero, 1998), y trata de determinar:

- Si continúa sin fumar.

- Si se ha recaído: circunstancias de la recaída, atribución de la misma e intención de volver a intentarlo.

- Si se mantiene sin fumar: si ha existido algún contacto esporádico, si ha sido ayudado, si ha variado su peso y si tiene intención de continuar sin fumar.

- Autoeficacia percibida en situaciones de riesgo de cara a mantenerse sin fumar.

\section{Análisis estadístico}

La determinación del valor predictivo de las distintas variables se realiza a través de un análisis bivariante, donde la variable dependiente es la abstinencia o no abstinencia de tabaco a los 6 meses de finalizado el tratamiento. Se utiliza Chi cuadrado para las varia- 
bles independientes cualitativas, y t de Student para las cuantitativas. Todos los análisis estadísticos se realizaron con el programa informático SPSS, versión 7.5.

\section{RESULTADOS}

Resumimos los resultados obtenidos en la tabla

Tabla 2. Resumen de resultados y significación estadística de las diferencias observadas a los 6 meses de seguimiento en las variables objeto de estudio.

\begin{tabular}{|c|c|c|c|c|c|c|c|}
\hline $\begin{array}{c}\text { TIPO DE } \\
\text { VARIABLE }\end{array}$ & VARIABLE & Valores & $\begin{array}{l}\text { Frecuencia } \\
\text { sí fuman a } \\
\text { los } 6 \text { meses }\end{array}$ & $\begin{array}{l}\text { Frecuencia } \\
\text { no fuman a } \\
\text { los } 6 \text { meses }\end{array}$ & $\begin{array}{c}\text { Media } \\
\text { grupo sí } \\
\text { fumadores }\end{array}$ & $\begin{array}{l}\text { Media } \\
\text { grupo no } \\
\text { fumadores }\end{array}$ & $\mathbf{p}$ \\
\hline \multirow{9}{*}{ 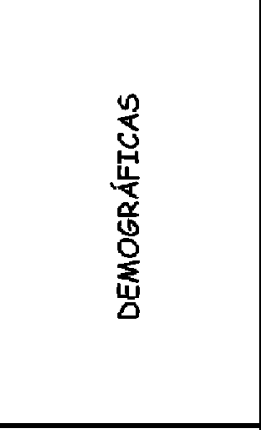 } & \multicolumn{4}{|l|}{ Edad } & 40,14 & 41,11 & $p=0,45$ \\
\hline & Sexo & Varón & $\begin{array}{l}13 \\
51\end{array}$ & $\begin{array}{l}26 \\
38\end{array}$ & & & $\mathrm{p}=0,01$ \\
\hline & & Tit. Superior & 21 & 22 & & & \multirow{5}{*}{$p=0,45$} \\
\hline & Grupo & Tit. Medio & 13 & 13 & & & \\
\hline & Thonfosinnal & FP2 o eq. & 0 & 2 & & & \\
\hline & \multirow{2}{*}{ profesional } & Fpl o eq. & 21 & 19 & & & \\
\hline & & Sin Titulación & $?$ & & & & \\
\hline & \multirow{2}{*}{ Vivir solo } & Vive solo & 8 & 9 & & & \multirow{2}{*}{$p=0,79$} \\
\hline & & No vive solo & 57 & 56 & & & \\
\hline \multirow{7}{*}{$\begin{array}{l}\text { HISTORIA DEL } \\
\text { CONSUMO }\end{array}$} & \multicolumn{4}{|c|}{ Semanas previas de abstinencia } & 23,66 & 21,84 & $p=0,83$ \\
\hline & \multicolumn{4}{|c|}{ Número de intentos previos } & 1,31 & 1,32 & $p=0,98$ \\
\hline & \multicolumn{3}{|l|}{ Años fumando } & & 21,46 & 22,52 & $p=0,4$ \\
\hline & \multicolumn{2}{|c|}{$\begin{array}{l}\text { Ningún fuma- } \\
\text { dor en casa }\end{array}$} & 11 & 15 & & & \multirow{4}{*}{$\mathrm{p}=0,45$} \\
\hline & \multirow{3}{*}{$\begin{array}{c}\text { fumadores en } \\
\text { casa }\end{array}$} & Un fumador en & 31 & 33 & & & \\
\hline & & \multicolumn{2}{|l|}{ Dos o más } & & & & \\
\hline & & $\begin{array}{l}\text { fumadores en } \\
\text { casa }\end{array}$ & 23 & 17 & & & \\
\hline & Puntuación tes & st de Fagerstrom & & & 5,34 & 4,82 & $p=0,24$ \\
\hline SEVERIDAD DE & Número de cig & garrillos/día & & & 27,56 & 25,71 & $p=0,38$ \\
\hline LA DEPENDENCIA & & Normal & 37 & 37 & & & \\
\hline & Marca & Light & 23 & 23 & & & $p=0,93$ \\
\hline & & Ultralight & 4 & 3 & & & \\
\hline EXPECTATIVAS & Al final del tra & atamiento & & & 78,41 & 85,72 & $p=0,14$ \\
\hline AUTOEFICACIA & A los 3 meses & & & & 68,42 & 85,77 & $\mathrm{p}=0,009$ \\
\hline "DESLICES" en I & las primeras & Sí & 36 & 4 & & & $P<001$ \\
\hline semano & & No & 29 & 61 & & & \\
\hline PATOLOGÍA & Patologías grav & & & & 0,67 & 0,94 & $p=0,21$ \\
\hline ASOCIADA & Patologías leve & & & & 3,4 & 3,03 & $p=0,44$ \\
\hline & Fase proceso & Preparación & 9 & 14 & & & \\
\hline & de cambio & Contemplación & 22 & 17 & & & $p=0,23$ \\
\hline MOTIVACIÓN & & Precontemplació & 5 & 2 & & & \\
\hline & & Preparación & 9 & 14 & & & $\mathrm{n}=0.12$ \\
\hline & & Resto & 27 & 19 & & & p-u,L \\
\hline & & Gana peso & 31 & 41 & & & \\
\hline & tratamiento & Pierde peso & 1 & 0 & & & $p=0,43$ \\
\hline AUMENTO DE & & Mantiene peso & 13 & 13 & & & \\
\hline PESO & & Gana peso & 9 & 20 & & & \\
\hline & A los 3 meses & Pierde peso & 1 & 1 & & & $p=0,6$ \\
\hline & & Mantiene peso & 8 & 10 & & & \\
\hline
\end{tabular}


Como puede observarse, sólo tres de las variables consideradas establecen diferencias significativas ( $p<$ $0,05)$ entre quienes continúan sin fumar y quienes recaen: el sexo (con mejor pronóstico los varones), las expectativas de autoeficacia medidas a los 3 meses de dejar de fumar sobre la capacidad para continuar sin fumar, y la existencia o no de algún "desliz" durante las primeras semanas de tratamiento. Los dos primeros factores se encuentran, además, relacionados: los varones puntúan significativamente más alto que las mujeres en autoeficacia percibida al final del tratamiento ( $p=0,038$; tabla 3 ). Estos resultados son consistentes, como veremos, con los encontrados en otros estudios, donde estos tres factores aparecen repetidamente como predictivos del resultado.

\begin{tabular}{|lcccc|}
\hline \multicolumn{3}{|c|}{$\begin{array}{l}\text { Tabla 3. Diferencias en la medida de la } \\
\text { autoeficacia entre varones y mujeres. }\end{array}$} \\
\hline & N & $\begin{array}{c}\text { MEDIA } \\
\text { AUTOEFICACIA }\end{array}$ & P \\
\hline SEXO & VARONES & 31 & 90,45 & 0,043 \\
& MUJERES & 74 & 81,04 & \\
\hline
\end{tabular}

Bordean la significación otras dos variables: las expectativas de autoeficacia al final del tratamiento ( $p$ $=0,14)$ y la fase del proceso de cambio según el modelo transteórico de Prochaska et al. (1993) ( $p=$ $0,12)$, que suelen mostrarse significativas en otros análisis.

Por el contrario, se encuentran lejos de mostrar diferencias significativas otros factores: la edad, el número y la duración de los intentos previos de abandonar el tabaco, los años de consumo, la severidad de la dependencia, el número de cigarrillos diario, la convivencia con otros fumadores, el vivir solo, la gravedad de la patología asociada y la ganancia de peso al dejar de fumar.

\section{DISCUSIÓN}

Conviene llamar la atención sobre dos condicionantes importantes de nuestro trabajo: la duración del período de seguimiento (6 meses) y las características de la muestra, ya descritas. Al tratarse de un grupo sano, es posible que el factor "patología asociada" sea escasamente relevante, lo cual no ocurriría si trabajáramos con individuos con seria patología cardiopulmonar; por otro lado, las condiciones de acceso al programa (gratuito, en horario de trabajo, etc.) tampoco imponen un filtro exigente en cuanto a la motivación previa; determinadas variables (como la "motivación") pueden verse afectadas.

\section{Sexo}

Nuestros resultados apoyan fuertemente $(p=0,01)$ las conclusiones de trabajos anteriores sobre el mejor pronóstico del fumador varón frente a la mujer (Nides et al., 1995; Swan et al., 1988; Norregaard et al., 1993; Bjornson et al., 1995; Mankani et al., 1996; Ward et al., 1997; Blake et al., 1989; Gourlay et al., 1994), si bien en otros trabajos no se encuentra este resultado (Matheny y Weatherman, 1998; Garvey et al., 1992; Vázquez y Becoña, 1998). En todo caso, como señalan Bjornson et al. (1995), no parece que sea el sexo "per se" el responsable de estas diferencias. En nuestra muestra encontramos diferencias significativas entre los dos sexos en la medida de la autoeficacia: la percepción de la propia capacidad para abandonar el tabaco es significativamente más reducida en el caso de las mujeres. Esta diferencia explicaría los peores resultados de las mujeres.

\section{Expectativas de Autoeficacia}

En nuestra investigación buscamos diferencias significativas en la medida de las expectativas de autoeficacia entre los sujetos que recayeron y los que no recayeron a los 6 meses de finalizado el tratamiento. Esta diferencia resultó no significativa, aunque se acerca, cuando se refiere a la medida de las expectativas de autoeficacia al final del tratamiento $(p=0,14)$, pero sí lo fue la medida tomada a los 3 meses de finalizado el tratamiento $(p=0,009)$. La medida de las expectativas de autoeficacia resulta, por lo tanto, predictiva del éxito únicamente cuando han transcurrido ya algunos meses sin fumar.

\section{Consumos aislados en las primeras semanas}

En nuestra investigación encontramos diferencias significativas $(p<0.05)$ entre abstinentes y no abstinentes a los 6 meses, entre quienes habían experimentado algún contacto con el tabaco en las primeras semanas y quienes no lo habían experimentado en absoluto. El 83, 3\% de quienes no habían fumado ningún cigarrillo ni calada durante las primeras semanas permanecían abstinentes a los 6 meses; sólo el $16,7 \%$ restante habían sido capaces de mantener la abstinencia después de algún cigarrillo o alguna calada en las primeras semanas tras dejar de fumar. En la construcción de las expectativas de autoeficacia, a las que nos referíamos anteriormente, parece que los primeros días tienen una importancia decisiva. Resulta crítico, por lo tanto, enfatizar ante los pacientes la importancia de la abstinencia absoluta en las dos primeras semanas, y tomar medidas para conseguirla. 


\section{Fase del proceso de cambio}

Nuestros datos, comparando la fase de "preparación" con el resto, no permiten considerar esta variable como predictiva, si bien se acerca ( $p=0.12$ ) y merece ser investigada más a fondo, tal vez mejorando los procedimientos de medida. En todo caso, es un problema frecuente en nuestra muestra: ofrecer a los participantes la posibilidad de participar en un programa para dejar de fumar totalmente gratuito (incluyendo parches), dentro del horario laboral, y junto con compañeros de trabajo facilita quizá "excesivamente" la participación y fomenta actitudes de "hacer la prueba, a ver qué pasa", sin tener suficientemente elaborada la decisión de dejar de fumar. No cabe duda de que el coste para los participantes (de dinero, de tiempo), habitual en otros programas, supone un "filtro motivacional" que tiene un reflejo en los resultados finales.

\section{Apoyo social y entorno de fumadores}

En nuestra investigación no se encuentran diferencias significativas entre los abstinentes y los no abstinentes a los 6 meses en cuanto a la presencia o no de fumadores en casa ( $p=0,45)$.

\section{Edad}

La variable edad, en nuestra muestra, no establece diferencias significativas ( $p=0,45)$. En todo caso, los sujetos de nuestra muestra están muy agrupados en torno a los 40 años (media 40,36, desviación típica 7,48 ) por lo que el grupo puede ser no representativo en esta variable.

\section{Aumento de peso}

A pesar de que la posible ganancia de peso es una de las dificultades más referidas para el mantenimiento de la abstinencia, no encontramos valor predictivo en la ganancia de peso durante las primeras semanas ni después de tres meses de finalizado el tratamiento ( $p=0,43$ y $p=0,6$ respectivamente). Tampoco lo encontramos en el grupo de mujeres $(p=0,55$ y $p=$ 0,3 para el aumento de peso al final del programa y a los 3 meses, respectivamente) a pesar de que son ellas las que refieren mayor preocupación por esta cuestión (Klesges y Klesges, 1988). Nuestros resultados coinciden con los de Pirie et al (1992), que tampoco encuentran que las mujeres que ganan más peso recaigan significativamente más que las que ganan menos peso. Probablemente la ganancia de peso en las primeras semanas es algo asumido, que el individuo espera que sea pasajero, y que se suele atribuir a cambios no permanentes en el metabolismo o en los hábitos. Por el contrario, seguir ganando peso meses después de haber dejado de fumar probablemente es algo ya no esperado y percibido como menos controlable y más duradero, lo cual favorecería el volver a fumar para controlar el peso.

\section{Otras variables}

Factores demográficos como el nivel profesional o el hecho de vivir solo no presentan ningún valor predictivo. Tampoco la severidad de la dependencia ni el tipo de tabaco que se consume. Asumíamos que el número de intentos previos y la duración de las abstinencias previas, por su previsible efecto sobre las expectativas de eficacia, podrían tener algún valor predictivo, pero no lo hemos encontrado. Finalmente, la presencia de complicaciones orgánicas asociadas al tabaco tampoco se muestra significativa, si bien hay que insistir en que nuestra muestra está formada, salvo excepciones, por individuos sanos y en edad laboral.

\section{CONCLUSIONES}

En nuestra investigación encontramos como variables con valor estadísticamente predictivo del éxito para dejar de fumar el sexo (mejor los varones), las expectativas de autoeficacia medidas a los 3 meses y el no cometer ningún "desliz" durante las primeras semanas. El porcentaje total de abstinentes a los 6 meses era el 50,2\% de nuestra muestra.

Por el contrario, no hemos encontrado valor predictivo en las variables de motivación, fase del proceso de cambio, entorno de fumadores, edad, aumento de peso, nivel profesional, puntuación en el test de Fagerstrom, número de cigarrillos fumados, número de intentos previos y duración de las abstinencias previas.

En general, del cotejo de nuestros datos con los de otras investigaciones se desprende la necesidad de continuar esta línea de investigación, particularmente sobre aquellas variables, señaladas en el texto, en las que existen resultados dispares.

Desde el punto de vista clínico, si la probabilidad de mantener la abstinencia está modulada por distintos factores individuales o contextuales, parece obligado apostar por un enfoque altamente individualizado en los tratamientos para dejar de fumar y, en general, para abandonar cualquier conducta adictiva. Se impone, a nuestro juicio, la necesidad de llevar a cabo, en cada caso, un análisis funcional de la conducta de fumar que considere las particulares situaciones estimulares asociadas al consumo (externas, sociales, emocionales, fisiológicas...), los particulares reforzadores de la conducta de fumar (positivos y negativos) y los particu- 
lares factores disposicionales (del individuo y de su entorno) que modifican la probabilidad de que se abandone el tabaco. Siempre con el fin de diseñar una estrategia a la medida del usuario. Cuando hablamos de los resultados a largo plazo (no lo olvidemos), quizá convendría sustituir la idea de que existen tratamientos más eficaces que otros, o de que existen individuos más resistentes que otros, por la idea de que los objetivos establecidos, o el abordaje diseñado para conseguirlos, se ha adecuado mejor o peor a las características de la conducta del individuo o del grupo particular sobre el que intervenimos.

Así, por ejemplo, en el caso de las diferencias de sexo, hay datos que apuntan, como hemos argumentado, a que en el caso de las mujeres es más probable que el papel regulador del tabaco sobre el estado de ánimo sea especialmente significativo, y por lo tanto que deba valorarse la inclusión en el tratamiento de técnicas para aprender a controlar el estado de ánimo y que promuevan el apoyo social. Existe cierta evidencia que muestra que las mujeres se benefician más de programas que incluyen apoyo social, mientras que los hombres tendrían ventajas en enfoques más individualistas. Por otro lado, las mujeres fumadoras manifiestan mayor preocupación por el posible aumento de peso a consecuencia del abandono del tabaco. Finalmente, las distintas fases del ciclo menstrual pueden contribuir a la diferencia en la experiencia del "craving" y por lo tanto en la probabilidad de la recaída (Hatsukami, 1998).

\section{BIBLIOGRAFÍA}

ANNIS, H. M. A relapse prevention model for treatment of alcoholics. En Blane, H., Leonard, K. (eds.). Psychological theories of drinking and alcoholism. New York. Guilford Press, 1986.

BAYOT, A., Y CAPAFONS, A. (1995). Diferencias individuales como predictores del éxito en la reducción del tabaquismo. Una revisión. Rev. Esp. Drogodep. 20 (1): 7-17.

BECOÑA, E. Tratamiento del tabaquismo. En Graña, J. L. : Conductas adictivas. Teoría, evaluación y tratamiento. Debate, 1994.

BECOÑA, E., FROJÁN, M. X., LISTA, M. J. (1988). Comparison between two self-efficacy scales in the maintenance of smoking cessation. Psychological Reports, 62: 359-362.

BJORNSON, W., RAND, C., CONNETT, J. E., LINDGREN, P., NIDES, M., POPE, F., BUIST, A., HOPPE-RYAN, C., O'HARA, P. (1995). Gender differences in smoking cessation after 3 years in the Lung Health Study. Am J Public Health. 85(2): 223-230.
BLAKE, S. M., KLEPP K. I., PECHACHEKT, F. FORSOM, A. R., LUEPKER, R. V., JACOBS, D. R., MITTERMARK, M. B. (1989). Differences in smoking cessation strategies between men and women. Addict. Behav. 14 (4): 409418.

BOLMAN, C., DE VRIES, H. (1998). Psycho-social determinants and motivational phases in smoking behavior of cardiac inpatients. Prev Med. 27(5 Pt 1): 738-747.

BOCK, B. C., GOLDSTEIN, M. G., MARCUS, B. H. (1996). Depression following smoking cessation in women. $\mathbf{J}$ Subst Abuse. 8(1): 137-144.

COHEN, S. Y LICHTENSTEIN, E. (1990). Perceived stress, quitting smoking and smoking relapse. Health Psychology, 9: 466-478.

COLLINS, R. L., EMONT, S. L., ZYWIAK, W. H. (1990). Social influence processes in smoking cessation: postquitting predictors of long-term outcome. J Subst Abuse. 2(4): 389-403.

COVEY, L. S., GLASSMAN, A. H., STETNER, F. (1990). Depression and depressive symptoms in smoking cessation. Compr Psychiatry. 31(4): 350-354.

CURRY, S., THOMPSON, B., SEXTON, M., OMENN, G. S. (1989). Psychosocial predictors of outcome in a worksite smoking cessation program. Am J Prev Med. 5(1): 2-7.

DAUGHTON, D.M., ROBERTS, D., PATIL, K. D., RENNARD, S. I. (1990). Smoking cessation in the workplace: evaluation of relapse factors. Prev Med. 19(2): 227-230.

DE VRIES, H., MUDDE, A.N., DIJKSTRA, A., WILLEMSEN, M. C. (1998). Differential beliefs, perceived social influences, and self-efficacy expectations among smokers in various motivational phases. Prev Med. 27(5 Pt 1): 681-689.

EPSTEIN, L. H., DICKSON, B.E., MCKENZIE, S., RUSSELL, P. O. (1984). The effect of smoking on perception of muscle tension. Psychofarmacology, 83

FARKAS, A. J., PIERCE, J. P., ZHU S. H., ROSBROOK, B., GILPIN, E. A., BERRY, C., KAPLAN, R. M. (1996). Addiction versus stages of change models in predicting smoking cessation. Addiction. 91(9): 1281-1292.

FIORE, M., NOVOTNY, T., PIERCE, J., GIOVINO, G., HATZIANDREU, E., NEWCOMB, P., SURAWICZ, T., DAVIS, R. (1990). Methods used to quit smoking in the United States. Do cessation programs help? $\mathbf{J}$ of the American Medical Association, 263: 2760-2765.

FROJÁN, M. X. (1991). Eficacia de las variables de control en un programa de tratamiento del hábito de fumar. Revista de Psicología de la Salud, 3(2): 133-153.

GARCÍA MERINO, I. Prevención del tabaquismo desde la Comunidad de Madrid. Comunicación Oral. Primeras Jornadas Municipales sobre prevención y tratamiento del Tabaquismo. Ayuntamiento de Madrid. 1998.

GARVEY, A. J., BLISS, R. E., HITCHCOCK, J. L., HEINOLD, J. W., ROSNER, B. (1992). Predictors of smoking relapse among self-quitters: a report from the normative aging study. Addict. Behav. 17 (4): 367-377.

GINSBERG, J. P., KLESGES, R. C., JOHNSON, K. C., ECK, L. H., MEYERS, A. W., WINDERS, S. A. (1997). The rela- 
tionship between a history of depression and adherence to a multicomponent smoking-cessation program. Addict Behav. 22(6): 783-787.

GOURLAY, S. G., FORBES, A., MARRINER, T., PETHICA, D., MCNEILL, J. J. (1994). Prospective study of factors predicting outcome of transdermal nicotine treatment in smoking cessation. BMJ Br Med 309(6958): 842847.

GROVE, J. R. (1993). Attributional correlates of cessation self-efficacy among smokers. Addict Behav. 18(3): 311-320.

GULLIVER, S. B., HUGHES, J. R., SOLOMON, J. L., DEY, A. N. (1995). An investigation of self-efficacy, partner support and daily stresses as predictors of relapse to smoking in self-quitters. Addiction. 90(6): 767-772.

HAAGA, D. A., STEWART, B. L. (1992). Self-efficacy for recovery from a lapse after smoking cessation. J Consult Clin Psychol. 60(1): 24-28.

HALL, S. M., MUÑOZ, R. F., REUS, V. I. Depression and smoking treatment: a clinical trial of an affect regulation treatment. In Problems of drug dependence: proceedings of the $52^{\text {nd }}$ Annual Scientific Meeting. NIDA, 1991.

HALL, S. M., MUÑOZ, R. F., REUS, V. I., SEES, K. L. (1993). Nicotine, negative affect and depression. J Cons. Clin. Psychology, 61: 761-767.

HATSUKAMI, D. K. Treatment of special populations. Addicted to Nicotine: A National Research Forum. National Institute on Drug Abuse. 1998.

HAVASSY, B.E., HALL, S. M., WASSERMAN, D. A. (1991). Social support and relapse: commonalities among alcoholics, opiate users, and cigarette smokers. Addictive Behaviors, 16: 235-246.

HERRERO, F.J., MORENO, J.J., RIVERO, A. Resultados del programa para dejar de fumar del Ayuntamiento de Madrid (en preparación).

HILL, H. A., SCHOENBACH, V. J., KLEINBAUM, D. G., STRECHER, V. J., ORLEANS, C. T., GEBSKI, V. J., KAPLAN, B. $H$. (1994). A longitudinal analysis of predictors of quitting smoking among participants in a self-help intervention trial. Addict Behav.19(2): 159-173.

HUGHES, J. R., GULLIVER, S. B., FENWICK, J. W., VALLIERE, W. A., CRUSER, K., PEPPER, S., SHEA, P., SOLOMON, L. J., FLYNN, B. S. (1992). Smoking cessation among self-quitters. Health Psychol. 11(5): 331-334.

KAMARCK, T. W., LICHTENSTEIN, E. (1988). Program adherence and coping strategies as predictors of success in a smoking treatment program. Health Psychol. 7(6): 557-574.

KENFORD, S. L., FIORE, M. C., JORENBY, D. E., SMITH, S. S., WETTER, D., BAKER, T. B. (1994). Predicting smoking cessation. Who will quit with and without the nicotine patch. JAMA. 271(8): 589-594.

KING, T. K., MARCUS, B. H., PINTO, B. M., EMMONS, K. M., ABRAMS, D. B. (1996). Cognitive-behavioral mediators of changing multiple behaviors: smoking and a sedentary lifestyle. Prev Med. 25(6): 684-691.
KLESGES, R. C., BROWN, K., PASCALE, R. W., MURPHY, M., WILLIAMS, E., CIGRANG, J. A. (1988). Factors associated with participation, attrition, and outcome in a smoking cessation program at the workplace. Health Psychol. 7(6): 575-589.

KLESGES, R. C., KLESGES, L. M. (1988). Cigarette smoking as a dieting strategy in a university population. Int $\mathbf{J}$ Eating Disord. 7: 423-429.

KOWALSKI, S. D. (1997). Self-esteem and self-efficacy as predictors of success in smoking cessation. J Holist Nurs. 15(2): 128-142.

LAZARUS, R. S., FOLKMAN, S. Estrés y procesos cognitivos. Martínez Roca, 1986.

MANKANI, S. K., GARABRANT, D. H., HOMA, D. M. (1996). Effectiveness of nicotine patches in a workplace smoking cessation program. An eleven-month follow-up study. J Occup Environ Med. 38(2): 184-189.

MARCUS, B. H., ALBRECHT, A. E., KING, T. K., PARISI, A. F., PINTO, B. M., ROBERTS, M., NIURA, R. S., ABRAMS, D. B. (1999). The efficacy of exercise as an aid for smoking cessation in women: a randomized controlled trial. Arch Intern Med. 159(11): 1229-1234.

MARCUS, B. H., ALBRECHT, A. E., NIAURA, R. S., TAYLOR, E. R., SIMKIN, L. R., FEDER, S. I., ABRAMS, D. B., THOMPSON, P. D. (1995). Exercise enhances the maintenance of smoking cessation in women. Addict Behav. 20(1): 87-92.

MARLATT, G. A., GORDON, J. R. Relapse prevention: maintenance strategies in the treatment of addictive behaviors. New York. Guilford Press, 1985.

MATHENY, K. B., WEATHERMAN, K. E. (1998). Predictors of smoking cessation and maintenance. J Clin Psychol. 54(2): 223-235.

MORENO, J. J., HERRERO, F.J. Tabaquismo. Programa para dejar de fumar. Díaz de Santos, 1998.

MOTHERSILL, K. J., MCDOWELL, I., ROSSER, W. (1988). Subject characteristics and long term post-program smoking cessation. Addict Behav. 13(1): 29-36.

MUÑOZ, R. F., MARTIN, B. V., POSNER, S. F., PEREZ, E. J. (1997). Mood management mail intervention increases abstinence rates for Spanish-speaking Latino smokers. Am J Community Psychol. 25(3): 325-343.

NIDES, M. A., RAKOS, R. F., GONZALES, D., MURRAY, R. P., TASHKIN, D. P., BJORNSON-BENSON, W. M., LINDGREN, P., CONNETT, J. E. (1995). Predictors of initial smoking cessation and relapse through the first 2 years of the Lung Health Study. J Consult Clin Psychol. 63(1): 60-69.

NORREGAARD, J., TONNESEN, P., PETERSEN, L. (1993). Predictors and reasons for relapse in smoking cessation with nicotine and placebo patches. Prev Med. 22(2): 261-271.

PINTO, B. M., BORRELLI, B., KING, T. K., BOCK, B. C., CLARK, M. M., ROBERTS, M., MARCUS, B. H. (1999). Weight control smoking among sedentary women. Addict Behav. 24(1): 75-86.

PIRIE, P. L., MCBRIDE, C. M., HELLERSTEDT, W., JEFFERY, R. W., HATSUKAMI, D., ALLEN, S., LANDO, H. (1992). 
Smoking cessation in women concerned about weight.

Am J Public Health, 82 (9): 1238-1243.

PROCHASKA, J.O., PROCHASKA, J. M. Modelo transteórico de cambio para conductas adictivas. En Casas, M. y Gossop, M. (eds.): Recaída y prevención de recaídas. CITRAN, 1993.

RICHMOND, R. L., AUSTIN, A., WEBSTER, I. W. (1988). Predicting abstainers in a smoking cessation programme administered by general practitioners. Int J Epidemiol. 17(3): 530-534.

SCHOLTE, R. H., BRETELER, M. H. (1997). Withdrawal symptoms and previous attempts to quit smoking: associations with self-efficacy. Subst Use Misuse. 32(2): 133-148.

SECKER-WALKER, R. H., FLYNN, B. S., SOLOMON, L. J., VACEK, P. M., BRONSON, D. L. (1990). Predictors of smoking behavior change 6 and 18 months after individual counseling during periodic health examinations. Prev Med. 19(6): 675-685.

STUART, K., BORLAND, R. MCMURRAY, N. (1994). Self-efficacy, health locus of control, and smoking cessation. Addict Behav. 19(1): 1-12.

SWAN, G. E., DENK, C. E., PARKER, S. D., CARMELLI, D., FURZE, C. T., ROSENMAN, R. H. (1988). Risk factors for late relapse in male and female ex-smokers. Addict Behav. 13(3): 253-266.
TALCOTT, G. W., FIEDLER, E. R., PASCALE, R.W., KLESGES, R. C., PETERSON, A. L., JOHNSON, R. S. (1995). Is weight gain after smoking cessation inevitable? J Consult Clin Psychol. 63(2): 313-316.

VÁZQUEZ, F. L. Y BECOÑA, E. (1998): Variables predictivas del consumo de cigarrillos y de la abstinencia en un programa para dejar de fumar. Psicología Conductual 6 (3): 501-518.

WARD, K. D., KLESGES, R. C., ZBIKOWSKI, S. M., BLISS, R. E., GARVEY, A. J. (1997). Gender differences in the outcome of an unaided smoking cessation attempt. Addict. Behav. 22 (4): 521-533.

WEST, R. J., HAJEK, P., BELCHER, M. (1989). Severity of withdrawal symptoms as a predictor of outcome of an attempt to quit smoking. Psychol Med. 19(4): 981-985.

WESTMAN, E. C., BEHM, F. M., SIMEL, D. L., ROSE, J. E. (1997). Smoking behavior on the first day of a quit attempt predicts long-term abstinence. Arch Intern Med. 157(3): 335-340.

WOJCIK, J. (1988). Social learning predictors of the avoidance of smoking relapse. Addictive Behaviors, 13: 177180.

ZIMMERMAN, R. S., WARHEIT, G. J., ULBRICH, P. M., AUTH, J. B. (1990). The relationship between alcohol use and attempts and success at smoking cessation. Addict Behav. 15(3): 197-207. 
